\title{
The Atom-Bond Connectivity Index of Catacondensed Polyomino Graphs
}

\author{
Jinsong Chen, ${ }^{1,2}$ Jianping Liu, ${ }^{1}$ and Qiaoliang $\mathrm{Li}^{2}$ \\ ${ }^{1}$ College of Mathematics and Computer Science, Fuzhou University, Fuzhou, Fujian 350108, China \\ ${ }^{2}$ College of Mathematics and Computer Science, Hunan Normal University, Changsha, Hunan 410081, China \\ Correspondence should be addressed to Jinsong Chen; fzu_cjs@126.com
}

Received 3 January 2013; Accepted 4 February 2013

Academic Editor: Zhengkun Huan

Copyright (c) 2013 Jinsong Chen et al. This is an open access article distributed under the Creative Commons Attribution License, which permits unrestricted use, distribution, and reproduction in any medium, provided the original work is properly cited.

Let $G=(V, E)$ be a graph. The atom-bond connectivity $(\mathrm{ABC})$ index is defined as the sum of weights $\left(\left(d_{u}+d_{v}-2\right) / d_{u} d_{v}\right)^{1 / 2}$ over all edges $u v$ of $G$, where $d_{u}$ denotes the degree of a vertex $u$ of $G$. In this paper, we give the atom-bond connectivity index of the zigzag chain polyomino graphs. Meanwhile, we obtain the sharp upper bound on the atom-bond connectivity index of catacondensed polyomino graphs with $h$ squares and determine the corresponding extremal graphs.

\section{Introduction}

One of the most active fields of research in contemporary chemical graph theory is the study of topological indices (graph topological invariants) that can be used for describing and predicting physicochemical and pharmacological properties of organic compounds. In chemistry and for chemical graphs, these invariant numbers are known as the topological indices. There are many publications on the topological indices, see [1-6].

Let $G=(V, E)$ be a simple graph of order $n$. A few years ago, Estrada et al. [7] introduced a further vertex-degreebased graph invariant, known as the atom-bond connectivity $(\mathrm{ABC})$ index. It is defined as:

$$
\operatorname{ABC}(G)=\sum_{u v \in E(G)} \sqrt{\frac{d_{u}+d_{v}-2}{d_{u} d_{v}}} .
$$

The $\mathrm{ABC}$ index keeps the spirit of the Randic index, and it provides a good model for the stability of linear and branched alkanes as well as the strain energy of cycloalkanes [7]. Recently, the study of the ABC index attracts some research attention $[6,8-12]$.
Polyomino graphs [13], also called chessboards [14] or square-cell configurations [15] have attracted some mathematicians' considerable attention because many interesting combinatorial subjects are yielded from them such as domination problem and modeling problems of surface chemistry. A polyomino graph [16] is a connected geometric graph obtained by arranging congruent regular squares of side length 1 (called a cell) in a plane such that two squares are either disjoint or have a common edge. The polyomino graph has received considerable attentions.

Next, we introduce some graph definitions used in this paper.

Definition 1 (see [4]). Let $G$ be a polyomino graph. If all vertices of $G$ lie on its perimeter, then $G$ is said to be catacondensed polyomino graph or tree-like polyomino graph. (see Figure 1).

Definition 2 (see [16]). Let $G$ be a chain polyomino graph with $h$ squares. If the subgraph obtained from $G$ by deleting all the vertices of degree 2 and all the edges adjacent to the vertices is a path, then $G$ is said to be the zigzag chain polyomino graph, denoted by $Z_{h}$ (see Figure 1).

In this paper, we give the $\mathrm{ABC}$ indices of the zigzag chain polyomino graphs with $h$ squares and obtain the sharp upper 
bound on the $\mathrm{ABC}$ indices of catacondensed polyomino graphs with $h$ squares and determine the corresponding extremal graphs.

\section{The ABC Indices of Catacondensed Polyomino Graphs}

Let

$$
\begin{aligned}
& S_{1}=\left\{\sqrt{2}+\frac{2}{3}, \sqrt{2}+\frac{\sqrt{15}}{3}-\frac{2}{3}, 2\right\}, \\
& S_{2}=\left\{2, \frac{3 \sqrt{2}}{2}+\frac{\sqrt{6}}{4}+\frac{\sqrt{15}}{3}-2,\right. \\
& \frac{4}{3}+\frac{\sqrt{15}}{6}, \frac{2}{3}+\frac{\sqrt{15}}{3}, \sqrt{2}+\frac{\sqrt{15}}{3}-\frac{2}{3} \\
& \sqrt{2}+\frac{\sqrt{6}}{4}+\frac{\sqrt{15}}{6}-\frac{2}{3}, \sqrt{2}+\frac{\sqrt{6}}{4}, \\
& \sqrt{2}+\frac{\sqrt{6}}{2}-\frac{\sqrt{15}}{6}, \frac{\sqrt{2}}{2}+\frac{\sqrt{6}}{4}+\frac{2}{3}, \\
& \frac{\sqrt{2}}{2}+\frac{\sqrt{6}}{4}+\frac{\sqrt{15}}{6}, \frac{\sqrt{2}}{2}+\frac{\sqrt{6}}{2}+\frac{2}{3}-\frac{\sqrt{15}}{6}, \\
& \frac{\sqrt{2}}{2}+\frac{\sqrt{6}}{4}+\frac{\sqrt{15}}{3}-\frac{2}{3}, \frac{\sqrt{2}}{2}+\frac{\sqrt{6}}{2}, \\
& \frac{3 \sqrt{2}}{2}+\frac{\sqrt{6}}{4}+\frac{\sqrt{15}}{2}-\frac{8}{3}, \frac{3 \sqrt{2}}{2}+\frac{2 \sqrt{6}}{4}+\frac{\sqrt{15}}{6}-2 \text {, } \\
& \frac{3 \sqrt{2}}{2}+\frac{\sqrt{6}}{4}+\frac{4 \sqrt{15}}{6}-\frac{10}{3} \\
& \frac{3 \sqrt{2}}{2}+\frac{\sqrt{6}}{2}+\frac{2 \sqrt{15}}{6}-\frac{8}{3}, \frac{3 \sqrt{2}}{2}+\frac{3 \sqrt{6}}{4}-2, \\
& \frac{3 \sqrt{2}}{2}+\frac{3 \sqrt{6}}{4}-\frac{\sqrt{15}}{3}-\frac{2}{3}, \frac{3 \sqrt{2}}{2}+\frac{3 \sqrt{6}}{4}-\frac{\sqrt{15}}{6}-\frac{4}{3} \text {, } \\
& \frac{3 \sqrt{2}}{2}+\sqrt{6}-\frac{\sqrt{15}}{2}-\frac{2}{3}, \frac{3 \sqrt{2}}{2}+\sqrt{6}-\frac{2 \sqrt{15}}{6}-\frac{4}{3}, \\
& \frac{3 \sqrt{2}}{2}+\frac{5 \sqrt{6}}{4}-\frac{2 \sqrt{15}}{3}-\frac{2}{3} \\
& \left.\frac{3 \sqrt{2}}{2}+\frac{\sqrt{6}}{2}-\frac{4}{3}, \frac{3 \sqrt{2}}{2}+\frac{3 \sqrt{6}}{4}+\frac{\sqrt{15}}{6}-\frac{8}{3}\right\},
\end{aligned}
$$$$
S=S_{1} \bigcup S_{2}
$$

We call $\sqrt{\left(d_{u}+d_{v}-2\right) / d_{u} d_{v}}$ the weight of the edge $u v$, denoted by $W_{u v}$.

Note that for any catacondensed polyomino graph $H^{*}$ with $h$ squares, it can be obtained by gluing a new square $s$ to some catacondensed polyomino graph $H$ with $h-1$ squares. So, we have the following lemma.

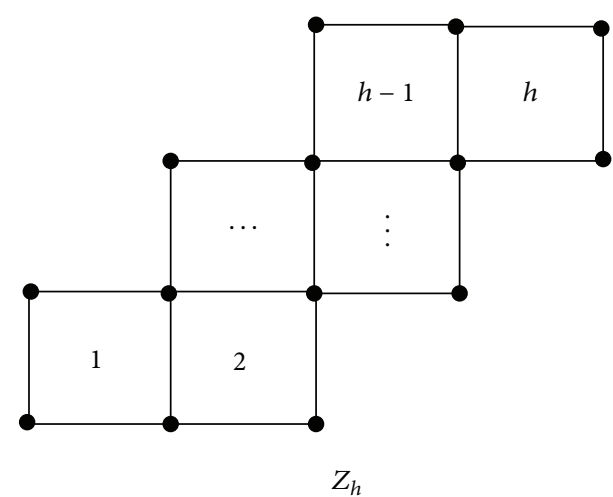

FIGURE 1: The zigzag chain polyomino graph $Z_{h}$.

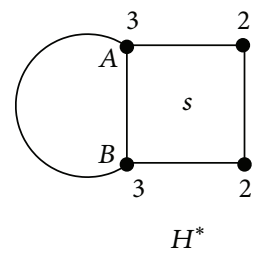

Type I

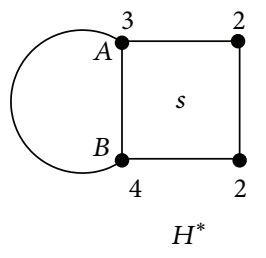

Type II

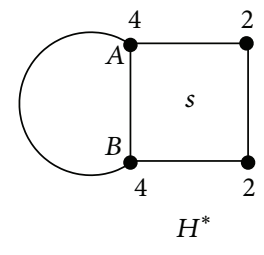

Type III
Figure 2

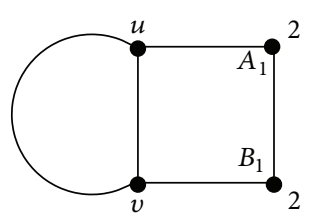

$H$

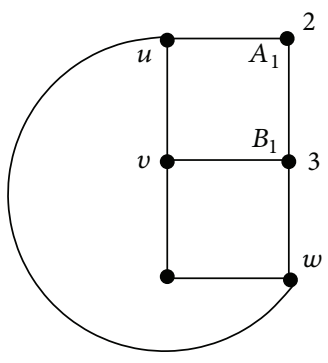

$H$

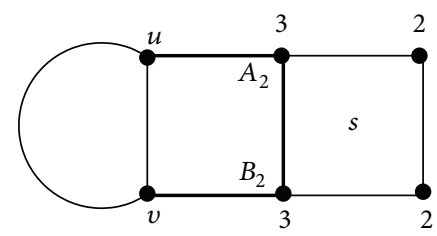

$H^{*}$
FIGURE 3

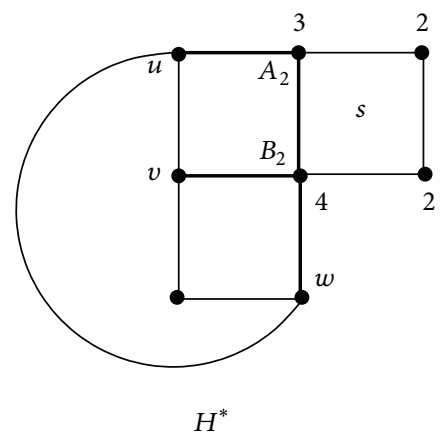

Figure 4

Lemma 3. Let $H^{*}$ be a catacondensed polyomino graph with $h$ squares which is obtained by gluing a new square s to some graph $H$, where $H$ is a catacondensed polyomino graph with $h-1$ squares. One has

(i) If $2 \leq h \leq 3$, then $A B C\left(H^{*}\right)-A B C(H) \in S_{1}$,

(ii) if $h \geq 4$, then $A B C\left(H^{*}\right)-A B C(H) \in S_{2}$. 


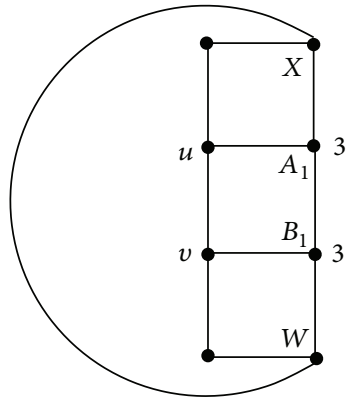

$H$

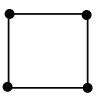

$H_{1}$

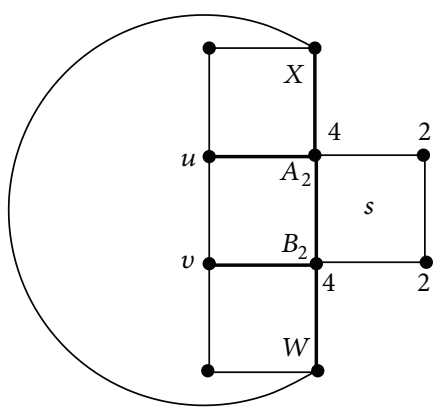

$H^{*}$
FIGURE 5

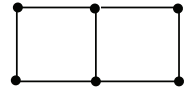

$\mathrm{H}_{2}$

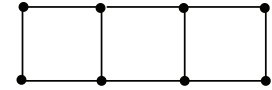

$\mathrm{H}_{3}$

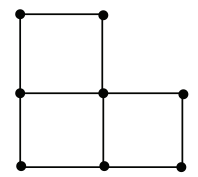

$\mathrm{H}_{4}$
FIGURE 6: A catacondensed polyomino graph with $h(h \leq 3)$ squares.

Proof. Consider the following: (i) if $2 \leq h \leq 3$, by directly calculating, we have $\operatorname{ABC}\left(H^{*}\right)-\operatorname{ABC}(H) \in S_{1}$,

(ii) now, let $h \geq 4$. Without the loss of generality, let square $s$ be adjacent to the edge $A B$ in $H$ (see Figure 2). In the following, if the weights of some edges of $H$ have been changed when $s$ is adjacent to the edge $A B$ in $H$, then we marked these edges with thick lines in $H^{*}$. Let $D_{i}=$ $\operatorname{ABC}\left(H^{*}\right)-\operatorname{ABC}(H)(i=1,2, \ldots, 35)$. Note that except the edge $A B$ of $s$, the summation of the weights of the remaining three edges is always $(3 / 2) \sqrt{2}$ in $H^{*}$. There are exactly three types of formations (see Figure 2).

Case 1. In Type I, $d_{A_{1}}=d_{B_{1}}=2$ and $d_{A_{2}}=d_{B_{2}}=3$ (see Figure 3).

By the definition of $\mathrm{ABC}$ index, we have $\mathrm{ABC}\left(H^{*}\right)-$ $\operatorname{ABC}(H)=(3 / 2) \sqrt{2}+\left(W_{u A_{2}}-W_{u A_{1}}\right)+\left(W_{A_{2} B_{2}}-W_{A_{1} B_{1}}\right)+$ $\left(W_{v B_{2}}-W_{v B_{1}}\right)=D_{i} \quad(i=1,2,3)$.

$$
\begin{aligned}
& \text { If } d_{u}=3 \text { and } d_{v}=3 \text {, then } D_{1}=2 \text {. } \\
& \text { If } d_{u}=3 \text { and } d_{v}=4 \text { or } d_{u}=4 \text { and } d_{v}=3 \text {, then } \\
& D_{2}=4 / 3+\sqrt{15} / 6 \text {. } \\
& \text { If } d_{u}=4 \text { and } d_{v}=4 \text {, then } D_{3}=2 / 3+\sqrt{15} / 3 \text {. }
\end{aligned}
$$

Case 2. In Type II, $d_{A_{1}}=2, d_{B_{1}}=3, d_{A_{2}}=3$, and $d_{B_{2}}=4$. (see Figure 4).

Let $u$ adjacent to $A$ and $v, w$ adjacent to $B$ (see Figure 4 ). Then $d_{u} \in\{2,3,4\}, d_{v} \in\{3,4\}$, and $d_{w} \in\{2,3,4\}$. If $d_{u}=2$, $d_{v}=3$, and $d_{w}=2$, which is in contradiction with $h \geq 4$; if $d_{u}=3$ and $d_{v}=3$, which is in contradiction with $d_{B_{1}}=3$; if $d_{u}=4$ and $d_{v}=3$, which is in contradiction with $d_{A_{1}}=2$ $(A \in V(H))$.

By the definition of $\mathrm{ABC}$ index, we have $\mathrm{ABC}\left(H^{*}\right)-$ $\operatorname{ABC}(H)=(3 / 2) \sqrt{2}+\left(W_{u A_{2}}-W_{u A_{1}}\right)+\left(W_{A_{2} B_{2}}-W_{A_{1} B_{1}}\right)+$ $\left(W_{v B_{2}}-W_{v B_{1}}\right)+\left(W_{w B_{2}}-W_{w B_{1}}\right)=D_{i}(i=4,5, \ldots, 14)$.
If $d_{u}=2, d_{v}=3$, and $d_{w}=3$, then $D_{4}=\sqrt{2}+$ $\sqrt{15} / 2-4 / 3$.

If $d_{u}=2, d_{v}=3$, and $d_{w}=4$, then $D_{5}=\sqrt{2}+\sqrt{6} / 4+$ $\sqrt{15} / 6-2 / 3$.

If $d_{u}=2, d_{v}=4$, and $d_{w}=2$, then $D_{6}=\sqrt{2}+\sqrt{6} / 4$.

If $d_{u}=2, d_{v}=4$, and $d_{w}=3$, then $D_{7}=\sqrt{2}+\sqrt{6} / 4+$ $\sqrt{15} / 6-2 / 3$.

If $d_{u}=2, d_{v}=4$, and $d_{w}=4$, then $D_{8}=\sqrt{2}+\sqrt{6} / 2-$ $\sqrt{15} / 6$.

If $d_{u}=3, d_{v}=4$, and $d_{w}=2$, then $D_{9}=\sqrt{2} / 2+$ $\sqrt{6} / 4+2 / 3$.

If $d_{u}=3, d_{v}=4$, and $d_{w}=3$, then $D_{10}=\sqrt{2} / 2+$ $\sqrt{6} / 4+\sqrt{5 / 12}$.

If $d_{u}=3, d_{v}=4$, and $d_{w}=4$, then $D_{11}=\sqrt{2} / 2+$ $\sqrt{6} / 2+2 / 3-\sqrt{5 / 12}$.

If $d_{u}=4, d_{v}=4$, and $d_{w}=2$, then $D_{12}=\sqrt{2} / 2+$ $\sqrt{6} / 4+\sqrt{5 / 12}$.

If $d_{u}=4, d_{v}=4$, and $d_{w}=3$, then $D_{13}=\sqrt{2} / 2+$ $\sqrt{6} / 4+2 \sqrt{5 / 12}-2 / 3$.

If $d_{u}=4, d_{v}=4$, and $d_{w}=4$, then $D_{14}=\sqrt{2} / 2+$ $\sqrt{6} / 2$.

Case 3. In Type III, $d_{A_{1}}=d_{B_{1}}=3$ and $d_{A_{2}}=d_{B_{2}}=4$ (see Figure 5).

Let $u, x$ adjacent to $A$ and $v, w$ adjacent to $B$ (see Figure 5). Then, $d_{u} \in\{3,4\}, d_{v} \in\{3,4\}, d_{w} \in\{2,3,4\}$, and $d_{x} \in$ $\{2,3,4\}$. Since the case $d_{u}=3, d_{v}=4, d_{x}=y_{1}$, and $d_{w}=y_{2}$ is the same as $d_{u}=4, d_{v}=3, d_{x}=y_{2}$, and $d_{w}=y_{1}$, where $y_{1}, y_{2} \in\{2,3,4\}$. And note that if $d_{u}=d_{v}=3$ or $d_{u}=d_{v}=4$, the vertices $x$ and $w$ are symmetric.

By the definition of $A B C$ index, we have $\operatorname{ABC}\left(H^{*}\right)-$ $\operatorname{ABC}(H)=(3 / 2) \sqrt{2}+\left(W_{x A_{2}}-W_{x A_{1}}\right)+\left(W_{u A_{2}}-W_{u A_{1}}\right)+$ $\left(W_{A_{2} B_{2}}-W_{A_{1} B_{1}}\right)+\left(W_{v B_{2}}-W_{v B_{1}}\right)+\left(W_{w B_{2}}-W_{w B_{1}}\right)=D_{i}$ $(i=15,16, \ldots, 35)$.

If $d_{u}=d_{v}=3, d_{x}=2$, and $d_{w}=3$, then $D_{15}=$ $3 \sqrt{2} / 2+\sqrt{6} / 4+\sqrt{15} / 2-8 / 3$

If $d_{u}=d_{v}=3, d_{x}=2$, and $d_{w}=4$, then $D_{16}=$ $3 \sqrt{2} / 2+\sqrt{6} / 2+\sqrt{15} / 6-2$.

If $d_{u}=d_{v}=3, d_{x}=3$, and $d_{w}=3$, then $D_{17}=$ $3 \sqrt{2} / 2+\sqrt{6} / 4+2 \sqrt{15} / 3-10 / 3$.

If $d_{u}=d_{v}=3, d_{x}=3$, and $d_{w}=4$, then $D_{18}=$ $3 \sqrt{2} / 2+\sqrt{6} / 2+\sqrt{15} / 3-8 / 3$.

If $d_{u}=d_{v}=3, d_{x}=4$, and $d_{w}=4$, then $D_{19}=$ $3 \sqrt{2} / 2+3 \sqrt{6} / 4-2$.

If $d_{u}=d_{v}=4, d_{x}=2$, and $d_{w}=2$, then $D_{20}=$ $3 \sqrt{2} / 2+3 \sqrt{6} / 4-\sqrt{15} / 3-2 / 3$.

If $d_{u}=d_{v}=4, d_{x}=2$, and $d_{w}=3$, then $D_{21}=$ $3 \sqrt{2} / 2+3 \sqrt{6} / 4-\sqrt{15} / 6-4 / 3$.

If $d_{u}=d_{v}=4, d_{x}=2$, and $d_{w}=4$, then $D_{22}=$ $3 \sqrt{2} / 2+\sqrt{6}-\sqrt{15} / 2-2 / 3$. 
If $d_{u}=d_{v}=4, d_{x}=3$, and $d_{w}=3$, then $D_{23}=$ $3 \sqrt{2} / 2+3 \sqrt{6} / 4-2$.

If $d_{u}=d_{v}=4, d_{x}=3$, and $d_{w}=4$, then $D_{24}=$ $3 \sqrt{2} / 2+\sqrt{6}-\sqrt{15} / 3-4 / 3$.

If $d_{u}=d_{v}=4, d_{x}=4$, and $d_{w}=4$, then $D_{25}=$ $3 \sqrt{2} / 2+5 \sqrt{6} / 4-2 \sqrt{15} / 3-2 / 3$.

If $d_{u}=3, d_{v}=4, d_{x}=2$, and $d_{w}=2$, then $D_{26}=$ $3 \sqrt{2} / 2+\sqrt{6} / 2-4 / 3$.

If $d_{u}=3, d_{v}=4, d_{x}=2$, and $d_{w}=3$, then $D_{27}=$ $3 \sqrt{2} / 2+\sqrt{6} / 2+\sqrt{15} / 6-2$.

If $d_{u}=3, d_{v}=4, d_{x}=2$, and $d_{w}=4$, then $D_{28}=$ $3 \sqrt{2} / 2+3 \sqrt{6} / 4-\sqrt{15} / 6-4 / 3$.

If $d_{u}=3, d_{v}=4, d_{x}=3$, and $d_{w}=2$, then $D_{29}=$ $3 \sqrt{2} / 2+\sqrt{6} / 2+\sqrt{15} / 6-2$.

If $d_{u}=3, d_{v}=4, d_{x}=3$, and $d_{w}=3$, then $D_{30}=$ $3 \sqrt{2} / 2+\sqrt{6} / 2+\sqrt{15} / 3-8 / 3$.

If $d_{u}=3, d_{v}=4, d_{x}=3$, and $d_{w}=4$, then $D_{31}=$ $3 \sqrt{2} / 2+3 \sqrt{6} / 4-2$.

If $d_{u}=3, d_{v}=4, d_{x}=4$, and $d_{w}=2$, then $D_{32}=$ $3 \sqrt{2} / 2+3 \sqrt{6} / 4-\sqrt{15} / 6-4 / 3$.

If $d_{u}=3, d_{v}=4, d_{x}=4$, and $d_{w}=3$, then $D_{33}=$ $3 \sqrt{2} / 2+3 \sqrt{6} / 4-2$.

If $d_{u}=3, d_{v}=4, d_{x}=4$, and $d_{w}=4$, then $D_{34}=$ $3 \sqrt{2} / 2+\sqrt{6}-\sqrt{15} / 3-4 / 3$.

If $d_{u}=3, d_{v}=3, d_{x}=2$, and $d_{w}=2$, then $D_{35}=$ $3 \sqrt{2} / 2+\sqrt{6} / 4+\sqrt{15} / 3-2$.

By directly calculating, we have $D_{5}=D_{7}, D_{10}=D_{12}$, $D_{16}=D_{27}=D_{29}, D_{18}=D_{30}, D_{19}=D_{23}=D_{31}=D_{33}$, $D_{21}=D_{28}=D_{32}, D_{24}=D_{34}$, and $D_{6}=\max _{1 \leq i \leq 35} D_{i}, D_{14}=$ $\min _{1 \leq i \leq 35} D_{i}$. So $\operatorname{ABC}\left(H^{*}\right)-\operatorname{ABC}(H) \in S_{2}$, where $h \geq 4$.

Therefore, $\mathrm{ABC}\left(H^{*}\right)-\mathrm{ABC}(H) \in S$.

By Lemma 3, we have the following theorem.
Theorem 4. Let $G$ be a catacondensed polyomino graph with $h(h \geq 2)$ squares, then

$$
\begin{aligned}
& A B C(G)=3 \sqrt{2}+\frac{2}{3}+\left(\sqrt{2}+\frac{\sqrt{15}}{3}-\frac{2}{3}\right) a_{1} \\
& +2 a_{2}+\left(\frac{4}{3}+\frac{\sqrt{15}}{6}\right) a_{3} \\
& +\left(\frac{2}{3}+\frac{\sqrt{15}}{3}\right) a_{4} \\
& +\left(\sqrt{2}+\frac{\sqrt{15}}{2}-\frac{4}{3}\right) a_{5} \\
& +\left(\sqrt{2}+\frac{\sqrt{6}}{4}+\frac{\sqrt{15}}{6}-\frac{2}{3}\right) a_{6} \\
& +\left(\sqrt{2}+\frac{\sqrt{6}}{4}\right) a_{7} \\
& +\left(\sqrt{2}+\frac{\sqrt{6}}{2}-\frac{\sqrt{15}}{6}\right) a_{8} \\
& +\left(\frac{\sqrt{2}}{2}+\frac{\sqrt{6}}{4}+\frac{2}{3}\right) a_{9} \\
& +\left(\frac{\sqrt{2}}{2}+\frac{\sqrt{6}}{4}+\frac{\sqrt{15}}{6}\right) a_{10} \\
& +\left(\frac{\sqrt{2}}{2}+\frac{\sqrt{6}}{2}+\frac{2}{3}-\frac{\sqrt{15}}{6}\right) a_{11} \\
& +\left(\frac{\sqrt{2}}{2}+\frac{\sqrt{6}}{4}+\frac{\sqrt{15}}{3}-\frac{2}{3}\right) a_{12} \\
& +\left(\frac{\sqrt{2}}{2}+\frac{\sqrt{6}}{2}\right) a_{13} \\
& +\left(\frac{3 \sqrt{2}}{2}+\frac{\sqrt{6}}{4}+\frac{\sqrt{15}}{2}-\frac{8}{3}\right) a_{14} \\
& +\left(\frac{3 \sqrt{2}}{2}+\frac{\sqrt{6}}{2}+\frac{\sqrt{15}}{6}-2\right) a_{15} \\
& +\left(\frac{3 \sqrt{2}}{2}+\frac{\sqrt{6}}{4}+\frac{2 \sqrt{15}}{3}-\frac{10}{3}\right) a_{16} \\
& +\left(\frac{3 \sqrt{2}}{2}+\frac{\sqrt{6}}{2}+\frac{\sqrt{15}}{3}-\frac{8}{3}\right) a_{17} \\
& +\left(\frac{3 \sqrt{2}}{2}+\frac{3 \sqrt{6}}{4}-2\right) a_{18} \\
& +\left(\frac{3 \sqrt{2}}{2}+\frac{3 \sqrt{6}}{4}-\frac{\sqrt{15}}{3}-\frac{2}{3}\right) a_{19}
\end{aligned}
$$




$$
\begin{aligned}
& +\left(\frac{3 \sqrt{2}}{2}+\frac{3 \sqrt{6}}{4}-\frac{\sqrt{15}}{6}-\frac{4}{3}\right) a_{20} \\
& +\left(\frac{3 \sqrt{2}}{2}+\sqrt{6}-\frac{\sqrt{15}}{2}-\frac{2}{3}\right) a_{21} \\
& +\left(\frac{3 \sqrt{2}}{2}+\sqrt{6}-\frac{\sqrt{15}}{3}-\frac{4}{3}\right) a_{22} \\
& +\left(\frac{3 \sqrt{2}}{2}+\frac{5 \sqrt{6}}{4}-\frac{2 \sqrt{15}}{3}-\frac{2}{3}\right) a_{23} \\
& +\left(\frac{3 \sqrt{2}}{2}+\frac{\sqrt{6}}{2}-\frac{4}{3}\right) a_{24} \\
& +\left(\frac{3 \sqrt{2}}{2}+\frac{\sqrt{6}}{4}+\frac{\sqrt{15}}{3}-2\right) a_{25}
\end{aligned}
$$

where $a_{i}$ is a nonnegative integer for $i=1,2, \ldots, 25$ and $h=$ $2+\sum_{i=1}^{25} a_{i}$.

Proof. We prove Theorem 4 by the induction on $h$. If $h=2$, by directly calculating, we have $\operatorname{ABC}(G)=3 \sqrt{2}+2 / 3$, where $a_{i}=0(i=1,2, \ldots, 25)$. So, Theorem 4 holds for $h=2$.

Assume that Theorem 4 holds for all catacondensed polyomino graphs with $h-1(h-1 \geq 2)$ squares, that is,

$$
\begin{aligned}
& \mathrm{ABC}(G)=3 \sqrt{2}+\frac{2}{3}+\left(\sqrt{2}+\frac{\sqrt{15}}{3}-\frac{2}{3}\right) a_{1} \\
& +2 a_{2}+\left(\frac{4}{3}+\frac{\sqrt{15}}{6}\right) a_{3} \\
& +\left(\frac{2}{3}+\frac{\sqrt{15}}{3}\right) a_{4} \\
& +\left(\sqrt{2}+\frac{\sqrt{15}}{2}-\frac{4}{3}\right) a_{5} \\
& +\left(\sqrt{2}+\frac{\sqrt{6}}{4}+\frac{\sqrt{15}}{6}-\frac{2}{3}\right) a_{6} \\
& +\left(\sqrt{2}+\frac{\sqrt{6}}{4}\right) a_{7} \\
& +\left(\sqrt{2}+\frac{\sqrt{6}}{2}-\frac{\sqrt{15}}{6}\right) a_{8} \\
& +\left(\frac{\sqrt{2}}{2}+\frac{\sqrt{6}}{4}+\frac{2}{3}\right) a_{9} \\
& +\left(\frac{\sqrt{2}}{2}+\frac{\sqrt{6}}{4}+\frac{\sqrt{15}}{6}\right) a_{10} \\
& +\left(\frac{\sqrt{2}}{2}+\frac{\sqrt{6}}{2}+\frac{2}{3}-\frac{\sqrt{15}}{6}\right) a_{11}
\end{aligned}
$$

$$
\begin{aligned}
& +\left(\frac{\sqrt{2}}{2}+\frac{\sqrt{6}}{4}+\frac{\sqrt{15}}{3}-\frac{2}{3}\right) a_{12} \\
& +\left(\frac{\sqrt{2}}{2}+\frac{\sqrt{6}}{2}\right) a_{13} \\
& +\left(\frac{3 \sqrt{2}}{2}+\frac{\sqrt{6}}{4}+\frac{\sqrt{15}}{2}-\frac{8}{3}\right) a_{14} \\
& +\left(\frac{3 \sqrt{2}}{2}+\frac{\sqrt{6}}{2}+\frac{\sqrt{15}}{6}-2\right) a_{15} \\
& +\left(\frac{3 \sqrt{2}}{2}+\frac{\sqrt{6}}{4}+\frac{2 \sqrt{15}}{3}-\frac{10}{3}\right) a_{16} \\
& +\left(\frac{3 \sqrt{2}}{2}+\frac{\sqrt{6}}{2}+\frac{\sqrt{15}}{3}-\frac{8}{3}\right) a_{17}
\end{aligned}
$$$$
+\left(\frac{3 \sqrt{2}}{2}+\frac{3 \sqrt{6}}{4}-2\right) a_{18}
$$$$
+\left(\frac{3 \sqrt{2}}{2}+\frac{3 \sqrt{6}}{4}-\frac{\sqrt{15}}{3}-\frac{2}{3}\right) a_{19}
$$$$
+\left(\frac{3 \sqrt{2}}{2}+\frac{3 \sqrt{6}}{4}-\frac{\sqrt{15}}{6}-\frac{4}{3}\right) a_{20}
$$$$
+\left(\frac{3 \sqrt{2}}{2}+\sqrt{6}-\frac{\sqrt{15}}{2}-\frac{2}{3}\right) a_{21}
$$$$
+\left(\frac{3 \sqrt{2}}{2}+\sqrt{6}-\frac{\sqrt{15}}{3}-\frac{4}{3}\right) a_{22}
$$$$
+\left(\frac{3 \sqrt{2}}{2}+\frac{5 \sqrt{6}}{4}-\frac{2 \sqrt{15}}{3}-\frac{2}{3}\right) a_{23}
$$$$
+\left(\frac{3 \sqrt{2}}{2}+\frac{\sqrt{6}}{2}-\frac{4}{3}\right) a_{24}
$$$$
+\left(\frac{3 \sqrt{2}}{2}+\frac{\sqrt{6}}{4}+\frac{\sqrt{15}}{3}-2\right) a_{25}
$$

where $a_{i}$ is a nonnegative integer for $i=1,2, \ldots, 25$ and $h-1=$ $2+\sum_{i=1}^{25} a_{i}$.

We will prove that Theorem 4 holds for $h$ in the following. Let $G^{*}$ be a catacondensed polyomino graph with $h$ squares. Without the loss of generality, $G^{*}$ can be obtained from some catacondensed polyomino graph $G$ with $h-1$ squares by gluing a new square $s$ to $G$. By Lemma 3, we have $\operatorname{ABC}\left(G^{*}\right)-$ $\mathrm{ABC}(G) \in S$. It means that $\operatorname{ABC}\left(G^{*}\right)=\operatorname{ABC}(G)+a$, where 

we have

$$
\begin{aligned}
& \operatorname{ABC}\left(G^{*}\right)=3 \sqrt{2}+\frac{2}{3}+\left(\sqrt{2}+\frac{\sqrt{15}}{3}-\frac{2}{3}\right) a_{1}^{*} \\
& +2 a_{2}^{*}+\left(\frac{4}{3}+\frac{\sqrt{15}}{6}\right) a_{3}^{*} \\
& +\left(\frac{2}{3}+\frac{\sqrt{15}}{3}\right) a_{4}^{*} \\
& +\left(\sqrt{2}+\frac{\sqrt{15}}{2}-\frac{4}{3}\right) a_{5}^{*} \\
& +\left(\sqrt{2}+\frac{\sqrt{6}}{4}+\frac{\sqrt{15}}{6}-\frac{2}{3}\right) a_{6}^{*} \\
& +\left(\frac{3 \sqrt{2}}{2}+\frac{3 \sqrt{6}}{4}-\frac{\sqrt{15}}{6}-\frac{4}{3}\right) a_{20}^{*} \\
& +\left(\frac{3 \sqrt{2}}{2}+\sqrt{6}-\frac{\sqrt{15}}{2}-\frac{2}{3}\right) a_{21}^{*} \\
& +\left(\frac{3 \sqrt{2}}{2}+\sqrt{6}-\frac{\sqrt{15}}{3}-\frac{4}{3}\right) a_{22}^{*} \\
& +\left(\frac{3 \sqrt{2}}{2}+\frac{5 \sqrt{6}}{4}-\frac{2 \sqrt{15}}{3}-\frac{2}{3}\right) a_{23}^{*} \\
& +\left(\frac{3 \sqrt{2}}{2}+\frac{\sqrt{6}}{2}-\frac{4}{3}\right) a_{24}^{*} \\
& +\left(\frac{3 \sqrt{2}}{2}+\frac{\sqrt{6}}{4}+\frac{\sqrt{15}}{3}-2\right) a_{25}^{*} .
\end{aligned}
$$$$
+\left(\sqrt{2}+\frac{\sqrt{6}}{4}\right) a_{7}^{*}
$$$$
+\left(\sqrt{2}+\frac{\sqrt{6}}{2}-\frac{\sqrt{15}}{6}\right) a_{8}^{*}
$$$$
+\left(\frac{\sqrt{2}}{2}+\frac{\sqrt{6}}{4}+\frac{2}{3}\right) a_{9}^{*}
$$$$
+\left(\frac{\sqrt{2}}{2}+\frac{\sqrt{6}}{4}+\frac{\sqrt{15}}{6}\right) a_{10}^{*}
$$$$
+\left(\frac{\sqrt{2}}{2}+\frac{\sqrt{6}}{2}+\frac{2}{3}-\frac{\sqrt{15}}{6}\right) a_{11}^{*}
$$$$
+\left(\frac{\sqrt{2}}{2}+\frac{\sqrt{6}}{4}+\frac{\sqrt{15}}{3}-\frac{2}{3}\right) a_{12}^{*}
$$$$
+\left(\frac{\sqrt{2}}{2}+\frac{\sqrt{6}}{2}\right) a_{13}^{*}
$$$$
+\left(\frac{3 \sqrt{2}}{2}+\frac{\sqrt{6}}{4}+\frac{\sqrt{15}}{2}-\frac{8}{3}\right) a_{14}^{*}
$$$$
+\left(\frac{3 \sqrt{2}}{2}+\frac{\sqrt{6}}{2}+\frac{\sqrt{15}}{6}-2\right) a_{15}^{*}
$$$$
+\left(\frac{3 \sqrt{2}}{2}+\frac{\sqrt{6}}{4}+\frac{2 \sqrt{15}}{3}-\frac{10}{3}\right) a_{16}^{*}
$$$$
+\left(\frac{3 \sqrt{2}}{2}+\frac{\sqrt{6}}{2}+\frac{\sqrt{15}}{3}-\frac{8}{3}\right) a_{17}^{*}
$$$$
+\left(\frac{3 \sqrt{2}}{2}+\frac{3 \sqrt{6}}{4}-2\right) a_{18}^{*}
$$$$
+\left(\frac{3 \sqrt{2}}{2}+\frac{3 \sqrt{6}}{4}-\frac{\sqrt{15}}{3}-\frac{2}{3}\right) a_{19}^{*}
$$

There exists some $l \in\{1,2, \ldots, 25\}$ such that $a_{l}^{*}=a_{l}+1$ and $a_{j}^{*}=a_{j}$ for $j \neq l(j \in\{1,2, \ldots, 25\})$. Obviously, $a_{i}^{*}$ is a nonnegative integer for $i=1,2, \ldots, 25$ and $2+\sum_{i=1}^{25} a_{i}^{*}=$ $2+1+\sum_{i=1}^{25} a_{i}=h$.

Lemma 5. Let $H$ be a catacondensed polyomino graph with $h$ squares. If $h \leq 3$, there are exactly four nonisomorphism catacondensed polyomino graphs (see Figure 6), where $A B C\left(H_{1}\right)=$ $2 \sqrt{2}, A B C\left(H_{2}\right)=3 \sqrt{2}+2 / 3, A B C\left(H_{3}\right)=3 \sqrt{2}+8 / 3$, $A B C\left(H_{4}\right)=4 \sqrt{2}+\sqrt{15} / 3$.

Theorem 6. Let $Z_{h}$ be a zigzag chain polyomino graph with $h$ squares, then

$$
A B C\left(Z_{h}\right)= \begin{cases}2 \sqrt{2}, & h=1, \\ 3 \sqrt{2}+\frac{2}{3}, & h=2, \\ (h+1) \sqrt{2}+(h-3) \cdot \frac{\sqrt{6}}{4}+\frac{\sqrt{15}}{3}, & h \geq 3 .\end{cases}
$$

Proof. Obviously, $Z_{h}$ can be obtained by gluing a new square $s_{h}$ to $Z_{h-1}$. Let $s_{h-1}$ be the square adjacent to $s_{h}$ (see Figure 1 ). We will prove Theorem 6 by the induction on $h$.

If $h=1,2,3$, then Theorem 6 holds (by Lemma 5). Assume that $\operatorname{ABC}\left(Z_{h-1}\right)=(h-1+1) \sqrt{2}+(h-1-3) \cdot(\sqrt{6} / 4)+$ $(\sqrt{15} / 3)=h \sqrt{2}+(h-4) \cdot(\sqrt{6} / 4)+(\sqrt{15} / 3)$ for $h-1 \geq 3$. By the induction assumption and the $D_{6}$ in Lemma 3 , we have

$$
\begin{aligned}
\operatorname{ABC}\left(Z_{h}\right) & =\operatorname{ABC}\left(Z_{h-1}\right)+D_{6} \\
& =h \sqrt{2}+(h-4) \cdot \frac{\sqrt{6}}{4}+\frac{\sqrt{15}}{3}+\left(\sqrt{2}+\frac{\sqrt{6}}{4}\right) \\
& =(h+1) \sqrt{2}+(h-3) \cdot \frac{\sqrt{6}}{4}+\frac{\sqrt{15}}{3} .
\end{aligned}
$$

So, Theorem 6 holds. 
Note that $D_{6}=\max _{1 \leq i \leq 35} D_{i}$ for $h \geq 4$ and by Lemma 5 , we obtain the following Theorem 7 .

Theorem 7. Let $G$ be a catacondensed polyomino graph with $h$ squares, then $A B C(G) \leq(h+1) \sqrt{2}+(h-3) \cdot(\sqrt{6} / 4)+(\sqrt{15} / 3)$, with the equality if and only if $G \cong Z_{h}$.

\section{Acknowledgments}

The project was supported by NSFC (no. 11071272; 11101086; 11101087) and the Foundation of Fuzhou University (no. 2012XQ-30).

\section{References}

[1] A. Behtoei, M. Jannesari, and B. Taeri, "Maximum Zagreb index, minimum hyper-Wiener index and graph connectivity," Applied Mathematics Letters, vol. 22, no. 10, pp. 1571-1576, 2009.

[2] K. C. Das, I. Gutman, and B. Furtula, "Survey on geometricarithmetic indices of graphs," MATCH Communications in Mathematical and in Computer Chemistry, vol. 65, no. 3, pp. 595-644, 2011.

[3] B. Liu and I. Gutman, "On general Randić indices," MATCH Communications in Mathematical and in Computer Chemistry, vol. 58, no. 1, pp. 147-154, 2007.

[4] B. Liu and Z. You, "A survey on comparing Zagreb indices," MATCH Communications in Mathematical and in Computer Chemistry, vol. 65, no. 3, pp. 581-593, 2011.

[5] J. Liu and B. Liu, "E-L equienergetic graphs," Communications in Mathematical and in Computer Chemistry, vol. 66, no. 3, pp. 971-976, 2011.

[6] B. Zhou and N. Trinajstic, "Further results on atom-bond connectivity index of trees," Chemical Physics Letters, vol. 455, pp. 120-123, 2008.

[7] E. Estrada, L. Torres, L. Rodriguez, and I. Gutman, "An atombond connectivity index: modelling the enthalpy of formation of alkanes," Indian Journal of Chemistry A, vol. 37, pp. 849-855, 1998.

[8] J. Chen and X. Guo, "Extreme atom-bond connectivity index of graphs," MATCH Communications in Mathematical and in Computer Chemistry, vol. 65, no. 3, pp. 713-722, 2011.

[9] J. Chen and X. Guo, "The atom-bond connectivity index of chemical bicyclic graphs," Applied Mathematics B, vol. 27, no. 2, pp. 243-252, 2012.

[10] J. Chen, J. Liu, and X. Guo, "Some upper bounds for the atom-bond connectivity index of graphs," Applied Mathematics Letters, vol. 25, no. 7, pp. 1077-1081, 2012.

[11] B. Furtula, A. Graovac, and D. Vukičević, "Atom-bond connectivity index of trees," Discrete Applied Mathematics, vol. 157, no. 13, pp. 2828-2835, 2009.

[12] R. Xing, B. Zhou, and Z. Du, "Further results on atom-bond connectivity index of trees," Discrete Applied Mathematics, vol. 158, no. 14, pp. 1536-1545, 2010.

[13] C. Berge, C. C. Chen, V. Chvátal, and C. S. Seow, "Combinatorial properties of polyominoes," Combinatorica, vol. 1, no. 3, pp. 217224, 1981.

[14] E. J. Cockayne, "Chessboard domination problems," Discrete Mathematics, vol. 86, no. 1-3, pp. 13-20, 1990.
[15] F. Harary and P. Mezey, "Cell-shedding transformations, equivalence relations, and similarity measures for square-cell configurations," International Journal of Quantum Chemistry, vol. 62, pp. 353-361, 1997.

[16] R. Chen, "Perfect matchings of generalized polyomino graphs," Graphs and Combinatorics, vol. 21, no. 4, pp. 515-529, 2005. 


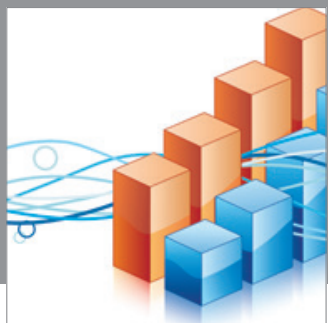

Advances in

Operations Research

mansans

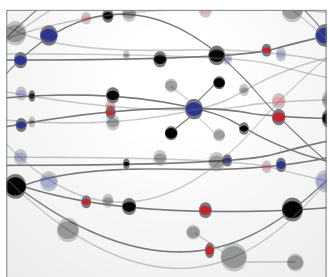

The Scientific World Journal
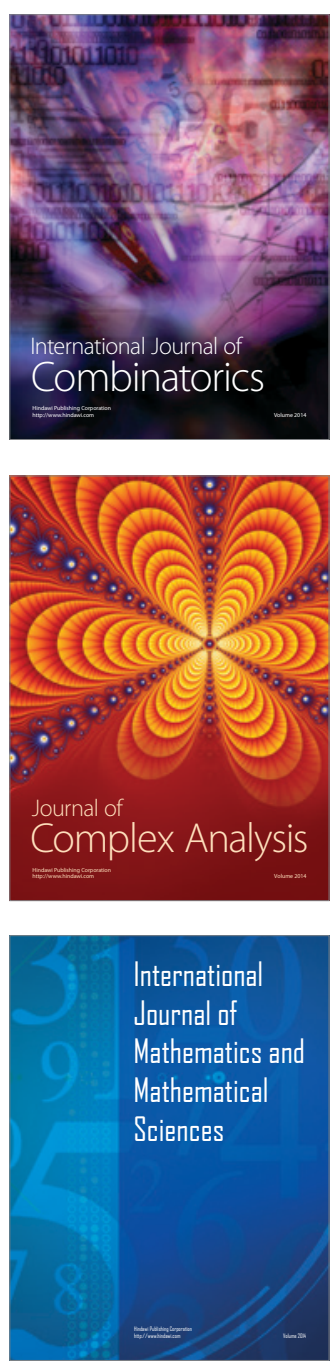
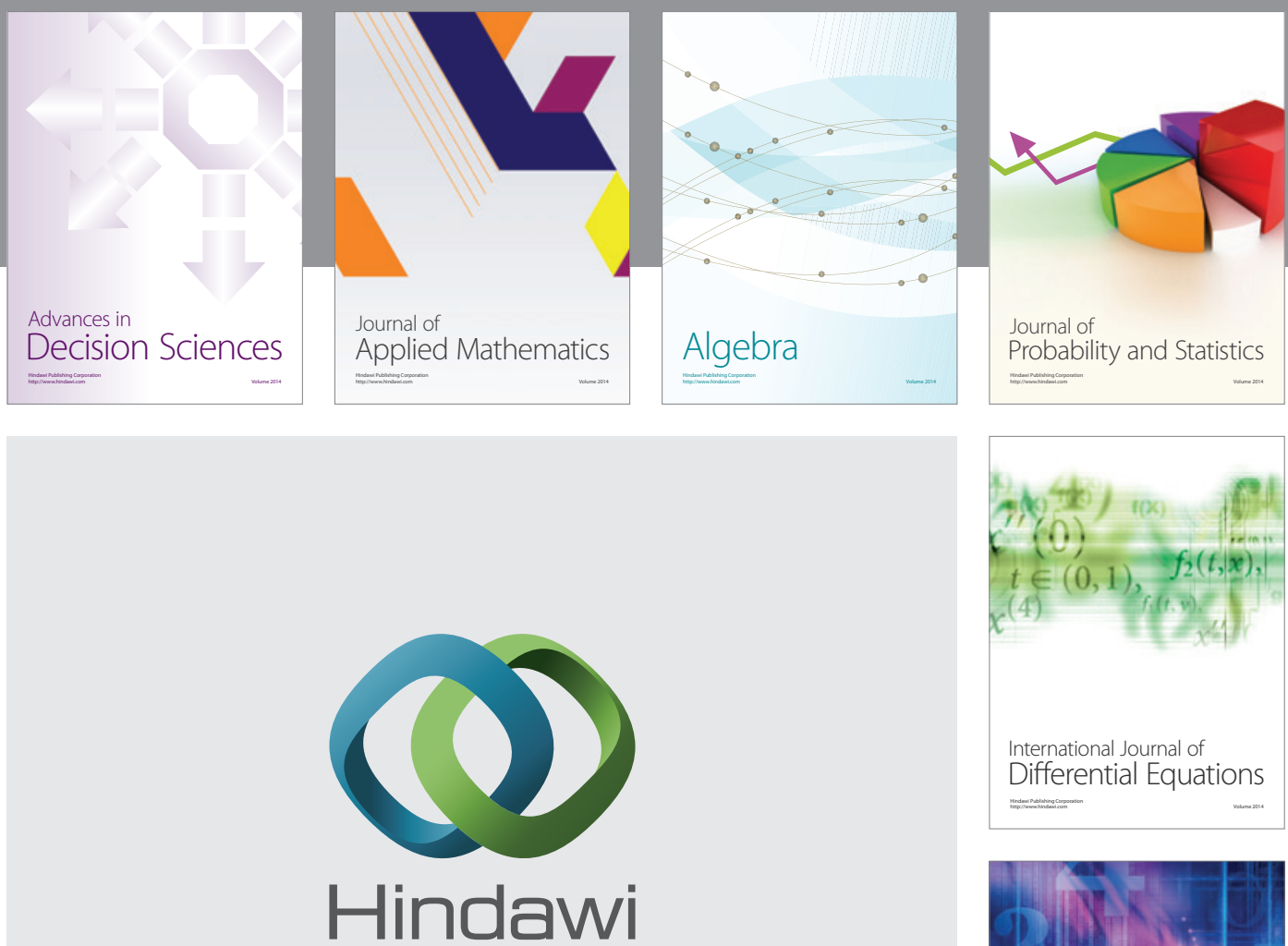

Submit your manuscripts at http://www.hindawi.com
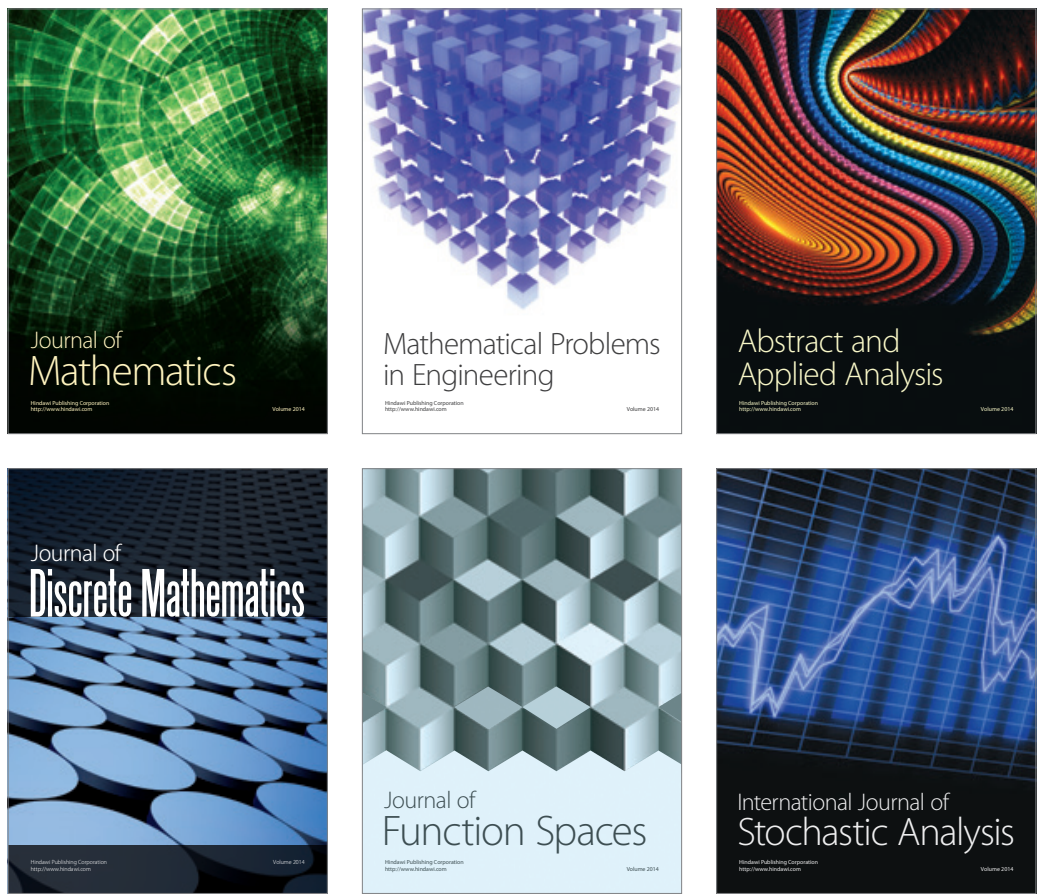

Journal of

Function Spaces

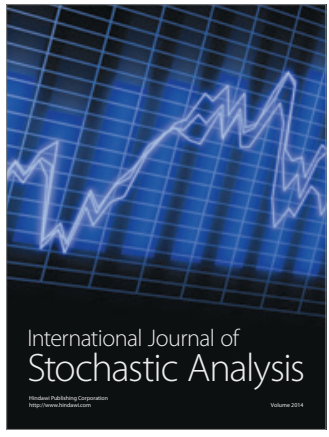

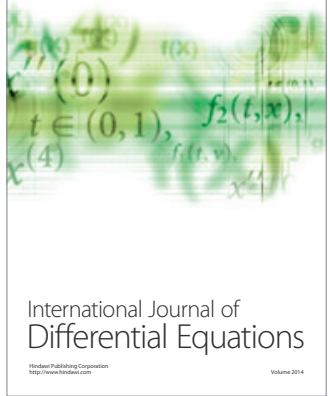
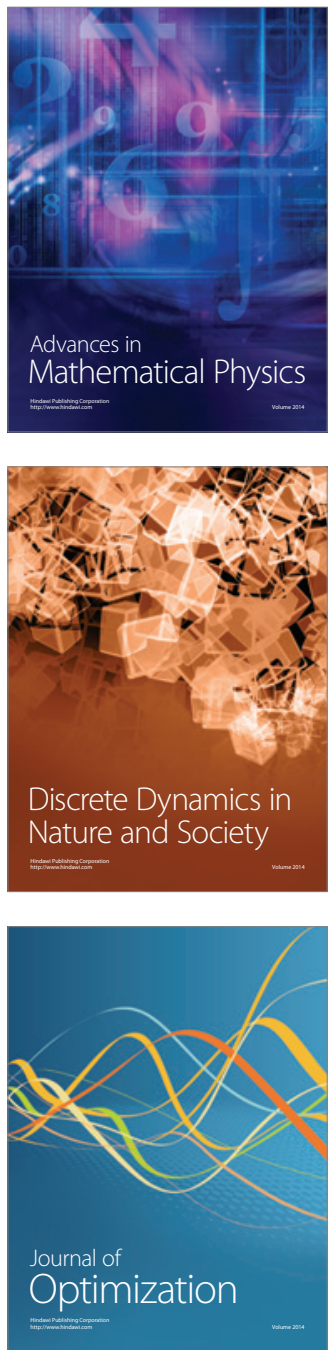